IDDF2018-ABS-0142 COMPARISON OF DIFFERENCES OF ESD WITH DIFFERENT DIAMETERS OF EARLY STAGE COLORECTAL CANCERS AND PRECANCEROUS COLORECTAL LESIONS

Hui Su*, Haihong Wang, Lili Liu, Tao Cheng, Yuqi He, Peng Jin, Lang Yang. PLA Army General Hospital, Beijing, China

\subsection{6/gutjnl-2018-IDDFabstracts. 125}

Background With the increase of diameters of colorectal tumours, procedure time of colorectal endoscopic submucosal dissection (CR - ESD) gradually prolonged. The aim of this study was to discuss the differences of ESD for colorectal tumours with different diameters, which was expected to provide some help for clinical work.

Methods This was a retrospective study of clinical data. From October 2012 to December 2015, we examined a total of 210 patients who were treated by an endoscopic procedure for colorectal tumours in the Endoscopy Centre, the PLA Army General Hospital. The lesions were divided into two groups according to different diameters $(\geq 4.0 \mathrm{~cm} \quad$ VS $<4.0 \mathrm{~cm}$ ) for comparative analysis of related factors.

Results The average procedure time of ESD of 210 colorectal tumour cases was $50.3 \pm 42.7 \mathrm{~min}$ and the average size was $7.98 \pm 10.84 \mathrm{~cm}^{2}$. En bloc resection rate was $91.4 \%$, R0 resection rate was $90.5 \%$, and the curative resection rate was $90.5 \%$. Perforation rate was $5.2 \%(11 / 210)$, and the late-onset haemorrhage rate was $0.5 \%(1 / 210)$. Compared with lesions with the diameters of $<4 \mathrm{~cm}$, tumours with diameters greater than $4 \mathrm{~cm}$ required longer resection time $(79.63 \pm 53.91 \mathrm{~min}$ vs $35.28 \pm 24.99 \mathrm{~min} ; \mathrm{p}<0.001)$; the lesions mainly located in the rectum (61.97\%); LST of mixed granular/nongranular type accounted for $54.93 \%$; en bloc resection rate, complete resection rate and curative resection rate of the tumours with diameters $\geq 4.0 \mathrm{~cm}$ was lower than those of $<4 \mathrm{~cm}$, the differences of complete resection rate was statistically significant $(85.92 \%$ vs. $94.24 \% ; \mathrm{p}=0.041)$. The perforation rate was higher $(7.04 \%)$ in the group with tumour sizes $\geq 4.0 \mathrm{~cm}$, but the difference was not statistically significant.

Conclusions ESDs of colorectal tumours with diameters greater than $4 \mathrm{~cm}$ require longer time, and the operation risk is higher. The operators should be more careful with non-rectal lesions.

\section{IDDF2018-ABS-0143 THE DIAGNOSIS AND TREATMENT OF PEUTZ-JEGHERS' SYNDROME}

Hui Su*, Yan Jia, Hao Fu, Na Li, Jianqiu Sheng. Department of Gastroenterology, PLA Army General Hospital, Beijing, China

\subsection{6/gutjnl-2018-IDDFabstracts.126}

Background To explore the clinical features, endoscope expression, pathology features, gene test results, diagnosis, treatment and prognosis of Peutz-Jeghers'syndrome (PJS).

Methods Retrospective analyses were performed on the clinical data of 46 cases of PJS patients in Department of Gastroenterology, PLA Army General Hospital from 2007 to 2017.

Results All 46 patients had mucocutaneous melanin pigmentation and multiple gastrointestinal polyposis. The pigmentation was first noticed often within $5 \mathrm{y}$, and 14 cases had a family history. The clinical manifestations were mainly black spots, abdominal pain, hematochezia and anaemia. Histological examinations showed that 20 patients were classified as hamartomatous polyps, 18 as adenomatous polyps, 14 as inflammatory polyps and 10 as zigzag polyps. The results of 11 patients sequenced a panel of 20 genes previously associated with CRC by next-generation sequencing showed 5 cases with gene mutations, and three of which with intussusception and surgical histories were found to have pathogenic germline mutations in the STK11 gene. Endoscopic treatment is the main therapy, but endoscopy combined with laparoscopy or surgical treatment should be available when complications occur, or the polyp is too large. A few patients found malignant tumours during follow-up.

Conclusions PJS is a hereditary disease which is characterised by spots of the skin or mucosa and gastrointestinal multiple polyps. The main pathological features were hamartoma and adenoma. The risk of intussusception and surgical operation is found to be high in the patients with pathogenic germline mutations in the STK11 gene, but the sample size was too small and needs to be further expanded. Endoscopic treatment is the main therapy. PJS patients should follow-up regularly due to the increased risk of cancer and being easy to relapse.

\section{IDDF2018-ABS-0145 COMPARATIVE ANALYSIS OF CLINICAL FEATURES OF CROHN'S DISEASE AND PRIMARY INTESTINAL LYMPHOMA}

Hui Su*, Yan Jia, Yuanming Pan, Limin Zhang, Shirong Li, Jianqiu Sheng. Department of Gastroenterology, PLA Army General Hospital, Beijing, China

\subsection{6/gutjnl-2018-IDDFabstracts. 127}

Background To compare the clinical characteristics of Crohn's disease (CD) and primary intestinal lymphoma (PIL), to find out valuable, relevant indicators for differential diagnosis.

Methods The clinical, endoscopic and pathological data of 67 cases (54 cases of Crohn's disease and 13 cases of primary intestinal lymphoma) admitted by the PLA Army General Hospital in the last seven years were analysed retrospectively.

Results

1. The average age of CD was lower than that of PIL, but the course of CD was longer than that of PIL $(p<0.01)$.

2. Abdominal pain, oral ulcers, perianal lesions, history of surgery (appendectomy or intestinal resection), and multi-site lesions were more common in CD than in PIL $(p<0.05)$. Abdominal mass, abdominal distension, single-site lesion was more common in patients with PIL than in CD $(p<0.05)$. The clinical manifestations of intestinal lymphoma were mostly mass type (53.8\%), and onsets in the ileocecal junction and terminal ileum were more common (69.2\%).

3. The biopsy diagnosis rate of PIL was $92.3 \%$, while $85.1 \%$ of CD's pathological manifestations were non-specific inflammation. So the biopsy diagnosis rate of PIL was significantly higher than that of CD $(p<0.05)$.

4. Two patients were initially diagnosed with $C D$ based on the clinical comprehensive analysis. The clinical symptoms disappeared, and the colonoscopy achieved mucosal healing after treatment. But the patients' symptoms repeated, and they were eventually diagnosed as PIL.

Conclusions The clinical and endoscopic characteristics of Crohn's disease (CD) and primary intestinal lymphoma (PIL) are similar, but they have their own characteristics. Therefore, the differential diagnosis is difficult. The diagnosis of CD is more supported by young, long course of the disease, abdominal pain, oral ulcers, perianal lesions, history of surgery (appendectomy, resection), and multi-site lesions. Short disease 